\title{
New strategies in behavioural phenotyping research: from individual domains to modeling domain networks
} Allan V Kalueff

\author{
Address: Laboratory of Clinical Science, National Institute of Mental Health, Bethesda, MD, USA \\ from International Society on Brain and Behaviour: 3rd International Congress on Brain and Behaviour \\ Thessaloniki, Greece. 28 November - 2 December 2007 \\ Published: 17 April 2008 \\ Annals of General Psychiatry 2008, 7(Suppl I):S26 doi:I0.II86/I744-859X-7-SI-S26
}

This abstract is available from: http://www.annals-general-psychiatry.com/content/7/SI/S26

(c) 2008 Kalueff; licensee BioMed Central Ltd.

Stress plays a key role in pathogenesis of anxiety and depression. Animal models of these disorders are widely used in behavioral neuroscience to explore stress-evoked brain abnormalities, screen anxiolytic/antidepressant drugs and establish behavioral phenotypes of gene-targeted or transgenic animals. Here we discuss the current situation with these experimental models, and critically evaluate the state of the art in this field. Noting a deficit of fresh ideas and especially new paradigms for animal anxiety and depression models, we review existing challenges and outline important directions for further research in this field. Potential strategies for the development of new animal paradigms include 1) modeling different subtypes of anxiety and depression, 2) their common pathogenesis, 3 ) the use of a wider spectrum of parameters, techniques and model objects. With psychiatric nomenclature and diagnostic criteria subject to constant modifications and reconsiderations, we may also benefit from 4 ) targeting a wider cluster of related behavioral phenomena (e.g., obsessive-compulsive disorders, Tourette's syndrome, addiction), 5) expanding models beyond traditional "anxiety" and "depression" domains, and 6) using "hybrid" models and tests. Together, these approaches will allow a better focus on the neurobiology of stress, enabling further integrative modeling of mood, behavioral and personality disorders consistent with recent trends and paradigmal shifts in modern psychiatry. One of the main reasons to invest time and efforts into new "integrative" models of anxiety and depression is the possibility to discover new agents or even principally new classes of psychotropic drugs, the need for which has long been recognized. In addition, this approach will increase our understanding of pathogenesis of anxiety and depression, and the link between these disorders and other brain illnesses. 